\title{
Intra operative assessment of the coronal balance in spinal deformity surgery : a technical note and retrospective study
}

\author{
Thibault Dewilde, Sebastiaan Schelfaut, Sven Bamps, Matthias Papen, Pierre Moens
}

From the University Hospitals Leuven, Leuven, Belgium

Obtaining a spine that is well balanced after fusion for scoliotic deformity is primordial for the patients' quality of life. A simple T-shaped instrument combined with standard intraoperative fluoroscopy can be of great help to evaluate the coronal alignment quickly. The aim of this study was to evaluate if a T-shaped device could predict the postoperative coronal balance. Before finalization of the rod fixation, the balance was checked by verifying the relationship between the $\mathrm{T}$-shaped instrument and the upper instrumented vertebra (UIV), and final adjustments were made to correct the coronal balance. A retrospective study was conducted on 48 patients who underwent surgery to correct scoliotic deformity. Intraoperative and postoperative coronal alignment was measured independently by two observers. The mean intraoperative horizontal offset measured between T-shaped instrument and the center of the UIV was $1,69 \mathrm{~mm}$ to the right with a standard deviation (SD) of $12,43 \mathrm{~mm}$. On postoperative full spine radiographs, the mean offset between the centra sacral vertical line and the center of the UIV was $2,44 \mathrm{~mm}$ to the left with a SD of $13,10 \mathrm{~mm}$. There is no significant difference in coronal balance between both measurements $(p=0,12)$. With this technique we were able to predict the postoperative coronal balance in all but one patient $(97,92 \%)$. We conclude that the use of a simple T-shaped instrument can provide adequate intraoperative assessment of coronal balance in correcting scoliotic deformity.

Keywords : scoliosis ; coronal balance ; intraoperative assesment ; coronal alignement ; scoliosis surgery.

Level of evidence : IV - case series

No benefits or funds were received in support of this study. None of the authors have a conflict of interest.

\section{INTRODUCTION}

Spinal balance is of great importance to spine surgeons. But more importantly, the patient benefits from a good coronal balance because it has a positive effect on the quality of life (1). Intra operative coronal balance assessment is often challenging due to patient positioning, patient draping and due to the limitations of fluoroscopy as it is difficult to capture the entire spinopelvic axis during deformity surgery. Guidelines for adequate coronal balance are scarce. A frequently used definition of coronal imbalance is a frontal shift of $>2 \mathrm{~cm}$, and an offset of $>4 \mathrm{~cm}$ in either direction should be avoided to prevent a significant decrease in functional outcome $(2,3)$. In 2012, Tolo et al reported on case examples of neuromuscular scoliosis patients who underwent deormity surgery in which a surgical technique, with a T-shaped instrument, was used to evaluate the coronal balance during surgery (4). In our

\footnotetext{
Thibault Dewilde, MD

Sebastiaan Schelfaut, MD

Sven Bamps, MD

Matthias Papen, MD

- Pierre Moens, MD

Spine Unit of the Orthopedic Department of the University Hospitals Leuven, Leuven, Belgium.

Correspondence: Thibault Dewilde, Dienst Orthopedie, Herestraat 49, 3000 Leuven, Belgium.

Email : thibault.dewilde@gmail.com

- 2021, Acta Orthopædica Belgica.
} 
department a similar technique has been used to address the same problem: verifying and guiding coronal balance during spinal deformity surgery. The aim of this study is to describe the surgical technique and analyzed it using a retrospective study design to demonstrate that, when using it correctly, outliers with significant postoperative coronal imbalance can be prevented.

\section{PATIENTS AND METHODS}

Medical records of 48 patients who underwent spinal deformity surgery were retrospectively evaluated. The T-shaped instrument was used in all cases so there was no need to limit eligibility with regards to age, sex, underlying disease or previous surgery. Only fixations reaching to the upper thoracic (T1-T4) region were included. The upper instrumented vertebra (UIV) was selected based on the curve flexibility, T1 tilt and shoulder balance.

All patients were operated in a prone position and intra operative and postoperative radiographic data were evaluated. The main object of the study is to evaluate if a correct use of the T-shaped device can predict postoperative coronal balance. To evaluate this, both the offsets between the center of the UIV and the T square instrument at this levels on intra operative images and between the center of the upper instrumented vertebra and the central sacral vertical line (CSVL) on the postoperative image were determined. All measurements were executed independently by 2 investigators. Since the fluoroscopic images are still measured in pixels, the width of the first thoracic vertebra (T1) was measured by the investigators between the tips of the transverse processes on both intra- and postoperative images to convert pixels into a metric range.

Guidelines for adequate coronal balance are scarce. A frequently used definition of coronal imbalance is a frontal shift of $>2 \mathrm{~cm}$ and an offset of $>4 \mathrm{~cm}$ in either direction. This should be avoided to prevent a significant decrease in functional outcome $(2,3)$. By default, a shift to the left of the CSVL is negative and positive when shifted to the right. Therefore, the aim of coronal balance correction should lie within a -2 to $+2 \mathrm{~cm}$ interval.

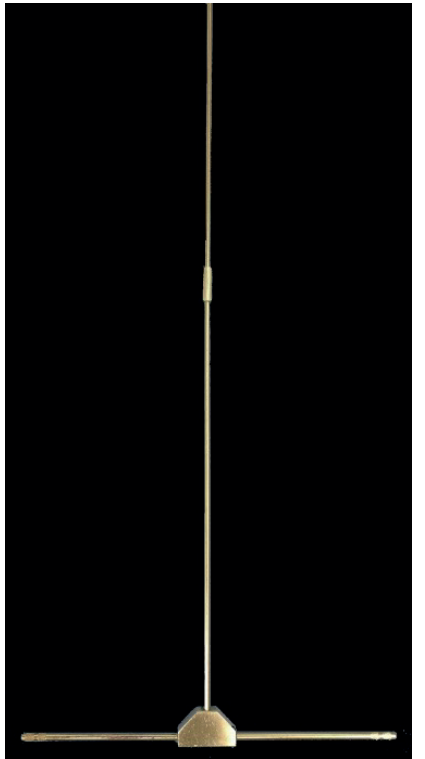

Figure 1. - T-shaped instrument.

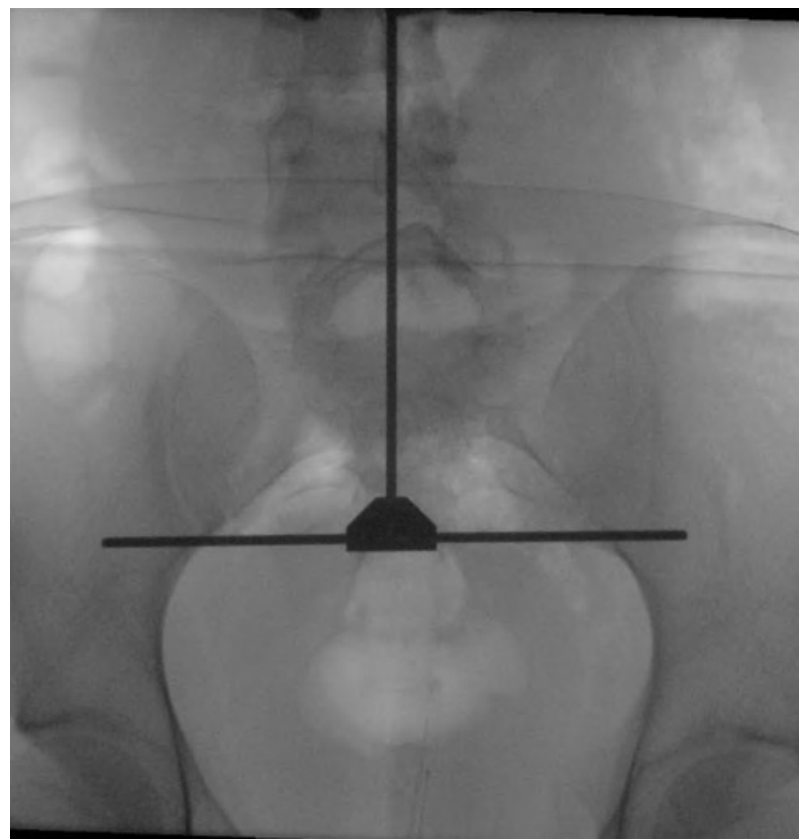

Figure 2. - Image of the instrument correctly positioned on the patients.

If the $\mathrm{T}$ square instrument is a reliable tool, intra operatively balanced patients should remain in balance postoperatively.

Statistical analyses were performed using IBM SPSS statistic release software. Interobserver reliability was calculated using the intraclass correlation 
(ICC) formula by Shrout and Fleis (5,6). Values of the ICC can vary from 0 to 1 with 1 indicating perfect reliability. ICC less than 0.4 indicate poor agreement ; 0.41 to 0.6 , moderate agreement ; 0.61 to 0.8 , good agreement and values greater than 0.8 excellent agreement (7). Further descriptive statistical analyses were used using mean values and standard deviations to show radiographic agreement between intra and postoperative imaging. Difference between the preoperative values and postoperative values were analyzed using a student t-test.

A radio opaque metal $\mathrm{T}$-shaped instrument (figure 1) is positioned on the pelvis and spine of the patient in prone position (figure 2). At the conclusion of the instrumentation and preliminary reduction an anteroposterior (AP) view of the pelvis in obtained. Care should be taken to have a perfect view of the pelvis : teardrops, iliac wings and obturator foramina should be symmetrical ; and if necessary the fluoroscopy should be adjusted. Then the T-shaped instrument is positioned with the horizontal limbs parallel to the pelvis connecting the inferior tip of both sacroiliac joints on fluoroscopic imaging. The vertical limb is then positioned in line with the central sacral vertical line. The fluoroscopy is then moved to the superior end of the construct to follow the vertical limb of the T-shaped instrument. The projection of the T-shaped instrument in relation with the upper instrumented vertebra allows for coronal balance estimation. Final adjustments were made to correct the coronal balance and was aimed to be between the margin of $2 \mathrm{~cm}$ left or right of the vertical limb.

The instrument is calibrated regularly to ensure a perfect 90 degree angle between the horizontal limbs and vertical limb.

\section{RESULTS}

A total of 48 patients, 12 males and 36 females, were withheld for inclusion with adequate imaging available. ICC $(3,1)$ for intraoperative offset measurements between the UIV and the T-shaped instrument device was 0,998 with a $95 \%$ confidence interval $(\mathrm{CI})$ of $[0,996-0,999]$, and 0,966 with a $95 \%$ CI of $[0,940-0,981]$ for postoperative UIV-CSVL offset measurements. D1 width measurements

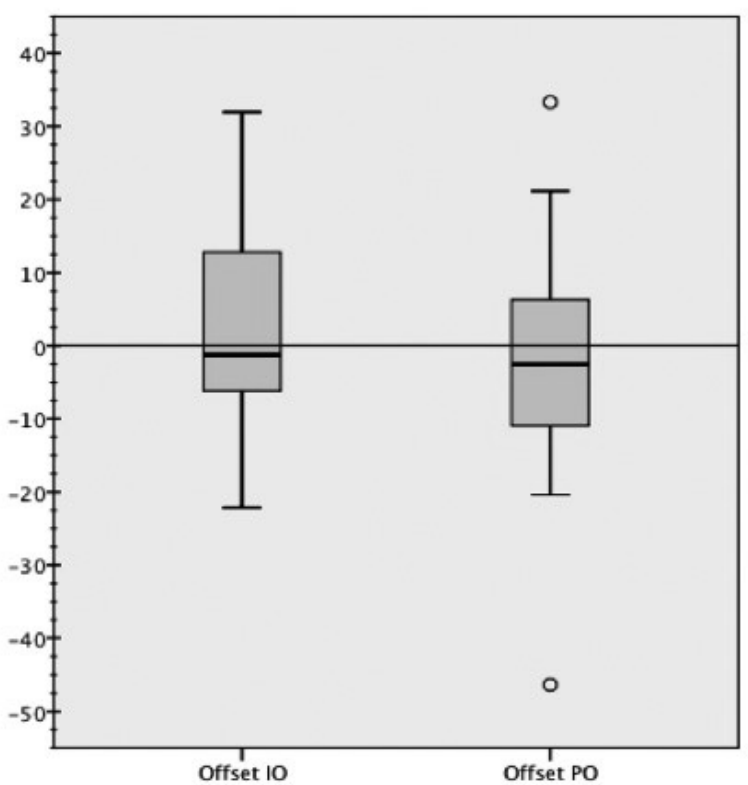

Figure 3. - Box-and-whisker plot showing average values for intraoperatievce (IO) and postoperative (PO) offset measurements in $\mathrm{mm}$.

correlated very closely between both observers. Intraoperative $\operatorname{ICC}(3,1)$ amounted to 0,998 with a $95 \%$ CI of $[0,997-0,999]$, postoperative $\operatorname{ICC}(3,1)$ was 0,997 with a $95 \%$ CI of $[0,995-0,998]$. Due to high ICC's between both intra- and postoperative D1-measurements, it was assumed appropriate to use these to convert all data to the metric system, making it easier to compare intra- and postoperative coronal balance measurements. After conversion, ICC $(3,1)$ for intraoperative offset measurements remained very high at 0,997 with a $95 \%$ CI of [0,995-0,999].

The average values of both observers' offset measurements were calculated. The mean intraoperative horizontal offset measured between the T-shaped instrument and the center of the UIV was $1,69 \mathrm{~mm}$ to the right with a standard deviation (SD) of $12,43 \mathrm{~mm}$. On postoperative full spine radiographs, the mean offset between the CSVL and the center of the UIV was $2,44 \mathrm{~mm}$ to the left with a SD of $13,10 \mathrm{~mm}$. The difference between the interoperative en postoperative measurements did not differ significantly $(\mathrm{p}=0.12)$. (figure 3 ) 
Out of 48 patients, four had an intraoperative coronal balance offset of $2-3 \mathrm{~cm}$ and only one had an offset of $3-4 \mathrm{~cm}$. Postoperatively, two patients measured a frontal shift of $2-3 \mathrm{~cm}$ with respect to the CSVL, one measured $3-4 \mathrm{~cm}$ and one had an offset of $>4 \mathrm{~cm}$. Two patients showed an interoperative slightly over $2 \mathrm{~cm}$, but were neutrally balanced postoperatively. Two others marginally crossed the $2 \mathrm{~cm}$ cutoff on postoperative imaging, but fell within the -2 to $2 \mathrm{~cm}$ interval intraoperatively. The average difference between intra- and postoperative offset measurements was $11,16 \mathrm{~mm}$.

\section{DISCUSSION}

Scoliosis causes a deformity in the sagittal and coronal plane. Compensatory curves often help to maintain a normal alignment. When correcting these deformities, a proper sagittal and coronal sagittal balance should be obtained. Especially the spinopelvic alignment, needed for a proper sitting balance, should be addressed with precision.

Obtaining a spine that is well balanced postoperatively is primordial for the patients' quality of life. Intra operative assessment is not always easy due to patient positioning, draping and the need for full spine radiographs.

Intraoperative assessment using a full spine radiograph is not readily available, requires special operating tables, exposes the patient to a higher dose of radiation and is time consuming. Therefore an alternative method is proposed. Only one simple T-shaped instrument is needed to evaluate the coronal alignment quickly. As this method is used before final fixation of the hardware, it can guide the surgeon to adjust the final coronal alignment. Tolo et al. were the first to describe a similar method in 2012 and some case examples were described (4). In 2018 Kurra et al used this method in 16 patients and compared the postoperative coronal alignment with 34 patients in which the instrument was not used (8). Patients were divided into two subgroups depending on the preoperative coronal malalignment ( $>20 \mathrm{~mm}$ and $>40 \mathrm{~mm}$ distance between C7PL and CSVL). A significantly greater correction of coronal malalignment and Cobb angles were observed in both subgroups when using the T-shaped instrument compared to not using the instrument.
Our study reports on the result of the use of a T-shaped instrument in 48 patients, the largest study group so far. The technique we used was slightly different to the ones previously described by the fact that the horizontal reference point for the pelvis is the lower sacroilliac joint and that the most cranial reference point was the UIV instead ofC 7 . We believe that the lower border of the sacroiliac joint provides a reproducible reference point and we choose the UIV because this is the highest reference point that can be influenced during finalization of the construction. With this technique we were able to predict the postoperative coronal balance in all but one patient $(97,92 \%)$. This patient was out of balance $>40 \mathrm{~mm}$ that was not predicted by the instrument. Retrospective evaluation of this case demonstrated an inadequate intraoperative positioning of the horizontal part of the instrument on the sacroiliac joint. Nevertheless a good overall alignment was obtained in this patient due to a compensatory lumbar curve. Retrospective analysis revealed inadequate positioning of the instrument in one more patient with on the intraoperative imaging an overestimation of $31,94 \mathrm{~mm}$ to the right. Postoperative imaging however showed a neutral coronal alignment. A third patient showed a positive shift tot the right of $33,30 \mathrm{~mm}$ that was already picked up intraoperatively by the instrument, but due to previous multilevel (L4-S1) interbody fusion further possibilities for coronal balance correction were limited and a slight undercorrection was accepted. The average difference between intra- and postoperative offset measurements of $11,16 \mathrm{~mm}$ can easily be attributed to positional dependency. When the patient is moved from prone surgical position into supine position in bed, a slight pelvic shift can cause an important offset variation at the level of the UIV. Nevertheless, $95 \%$ of intraoperatively neutrally balanced patients using the instrument remained within the -2 to $2 \mathrm{~cm}$ interval on immediate postoperative full spine radiographs. These minor coronal balance shifts are very often compensated postoperatively by non-fused segments as seen on standing full spine imaging taken during followup. 


\section{CONCLUSION}

We conclude that the use of a simple T-shaped instrument can provide adequate intraoperative assessment of coronal balance in correcting scoliotic deformity. Postoperative coronal balance can be predicted by the use of this instrument but compensatory curves of the non fused vertebrae are not totally accounted for. Further follow-up studies should be performed to evaluate the evolution of the coronal alignment in these patients at regular follow-up periods.

\section{REFERENCES}

1. Wai EK, Young NL, Feldman BM, et al. The Relationship Between Function, Self-perception, Implications for Treatment of Scoliosis in Children With Spina Bifida. J. Pediatr. Orthop. $2005 ; 25: 64-69$.

2. Gupta MC, Boachie-Adjei O, Cunningham ME, et al. Coronal imbalance may be neglected in patients undergoing major sagittal deformity correction. Spine J. 2013 ; 13 : 9S.
3. Malfair D, Flemming AK, Dvorak MF, et al. Radiographic evaluation of scoliosis: Review. Am. J. Roentgenol. 2010 ; 194 : 8-22.

4. Andras L, Yamaguchi KT, Skaggs DL, et al. Surgical technique for balancing posterior spinal fusions to the pelvis using the T square of tolo. $J$. Pediatr. Orthop. 2012 ; 32 : 63-66.

5. Rankin G, Stokes M. Reliability of assessment tools in rehabilitation: an illustration of appropriate statistical analyses. Clin. Rehabil. 1998; 12 : 187-99.

6. Shrout, Patrick E. Fleiss JL. Intraclass correlations: Uses in assessing rater reliability. Psychol. Bull. 1979 ; 86 : 420-428.

7. Majdouline Y, Aubin C-E, Robitaille M, et al. Scoliosis Correction in Adolescent Idiopathic Scoliosis. J. Pediatr Orthop. 2007 ; 27 : 775-781.

8. Kurra S, Metkar U, Yirenkyi $H$, et al. Assessment of Coronal Spinal Alignment for Adult Spine Deformity Cases After Intraoperative T Square Shaped Use. Spine Deform. 2018 ; 6 : 267-272. 